"The submitted manuscript has been authored by a contractor of the U.S. Government under contract No. DE-AC05-840R21400. Accordingly, the U.S.

Government retains a nonexclusive royalty-free

license to publish or reproduce the published form of

this contribution, or allow others to do so, for U.S.

Govemment purposes."

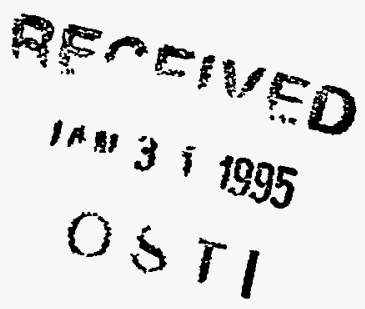

\title{
The dynamics of marginality and self-organized criticality as a paradigm for turbulent transport
}

\author{
D. E. Newman and B. A. Carreras
}

Oak Ridge National Laboratory, P.O. Box 2009, Oak Ridge, Tennessee 37831-8070

P. H. Diamond

University of California at San Diego, La Jolla, California 92093-0319

T. S. Hahm

Princeton Plasma Physics Laboratory, P.O. Box 451, Princeton, New Jersey 08543

\section{Abstract}

A general paradigm, based on the concept of self-organized criticality (SOC), for turbulent transport in magnetically confined plasmas has been recently suggested as an explanation for some of the apparent discrepancies between most theoretical models of turbulent transport and experimental observations of the transport in magnetically confined plasmas. This model describes the dynamics of the transport without relying on the underlying local fluctuation mechanisms. Computations based on a cellular automata realization of such a model have found that noise driven SOC systems can maintain average profiles that are linearly stable (submarginal) and yet are able to sustain active transport dynamics. It is also found that the dominant scales in the transport dynamics in the absence of sheared flow are system scales rather than the underlying local fluctuation scales. The addition of sheared flow into the dynamics leads to a large reduction of the system-scale transport events and a commensurate increase in the fluctuation-scale transport events 
needed to maintain the constant flux. The dynamics of these models and the potential ramifications for transport studies are discussed.

PACS: ????????? 


\section{INTRODUCTION}

Anomalous transport in magnetic confinement devices has defied simple characterization due in part to the observation that the dominant transport scale lengths have scaled with machine size (Bohm or worse scalings), while the suspected transport mechanisms have much smaller scales (Gyro-Bohm scaling). To shed some light on this apparent discrepancy and to investigate the effect of sheared flow on the transport dynamics and these scalings, a new approach to transport has been suggested ${ }^{1}$ based on the idea of self-organized criticality (SOC). ${ }^{2,3,4}$ This concept seeks to describe the dynamics of the transport without relying on the underlying local fluctuation mechanisms. Because of the independence of the transport dynamics on the specific local instability, this has the advantage of being more universally applicable and addresses some of the universal features such as profile robustness. The dynamics of such systems can be computationally investigated with a cellular automata model of "running sandpile" dynamics. This model allows us to investigate the major dynamical scales and the effect of an applied sheared flow on these dominant scales. A correspondence between many of the important quantities in turbulent transport and the equvilent variables in the SOC model is given in Table 1.

Two of the barriers in the way of understanding anomalous transport are (1) the questions of which instabilities are responsible for the transport and (2) the issue of the transport scale being reconciled with the fluctuation scale. It has long been believed that some linear instability (mode) is driving turbulent fluctuations, which are causing the anomalous transport. 5 A number of instabilities have been put forward as candidates for dominating transport in magnetic confinement devices. In many of these modes, a linear marginal stability condition has been assumed for the profile. This is based on the assumption that the turbulent system would relax its driving gradient back to the linearly least unstable profile (the marginal profile), just allowing for the drive to continue. Ballooning modes near the $\beta$ limit $^{6}$ are among the modes for which this has been suggested. In addition, ion temperature-gradient-driven modes at the marginal limit have 
been suggested as the dominant core transport mechanism.7,8 Unfortunately, all of these instability mechanisms suffer from the drawback that, experimentally, the profiles seem to be stable to the candidate modes over much if not all of the radius. ${ }^{9}$ The second difficulty is related to the fact that the transport from most of these modes is governed by the fluctuation scales, which are typically on the order of ion gyro radii (Gyro-Bohm scaling). ${ }^{10}$ These fluctuation scales define the characteristic "step size" of the turbulent diffusion leading to a confinement time that scales with the step size. Once again the experimental evidence is that the confinement in real magnetic confinement devices, at least in the low confinement mode (L-mode), scales with the machine size (Bohm scaling) ${ }^{\text {io }}$ rather than with the theoretical fluctuation step size. Interestingly, there is evidence that in the enhanced confinement modes ( $\mathrm{H}$ mode, etc.), which have a sheared flow coincident with the transport barrier, the confinement scaling seems to go from Bohm-like to GyroBohm-like. ${ }^{11}$

The major results in this paper are summarized below. It has been found that noise driven SOC systems maintain average profiles that are linearly stable (submarginal) and yet are able to sustain active transport dynamics in contrast to naive marginal stability arguments. This transport can occur on very fast time scales exhibiting etither very fast diffusive transport or even ballistic propagation. It is also found that with no sheared flow or sheared flow decorrelation times smaller than the natural system decorrelation times the dominant scales in the transport dynamics are system scales rather than the underlying local fluctuation scales. However, the addition of sheared flow into the dynamics leads to a large reduction of the system scale transport and a commensurate increase in the fluctuation scale transport. This may be consistent with the transition from Bohm to Gyro-Bohm scaling observed in improved confinement modes. Analytics on the Burgers equation ${ }^{1}$ show this transformation with the addition of shear as this as a change in the transport propagation scaling exponent with the propagation going from ballistic without shear to diffusive with shear. 
The remainder of the paper is organized as follows: Section II is a brief discussion of the heuristics of sheared flow in turbulent systems. Section III contains the SOC model investigated and the results from simulations without sheared flow. This is followed by Section IV, consisting of the results due to the addition of sheared flow to the SOC system. Finally, Section V is the conclusion and summary.

\section{HEURISTICS OF SHEARED FLOW IN TURBULENT SYSTEMS}

Due to in part the existence of a shear flow region coincident with the transport barrier in enhanced confinement modes, there has been much interest recently in the effect of shear flows on turbulent systems. This interaction can take a number of forms. The first and most often quoted is the shear suppression of the turbulence. ${ }^{12}$ This occurs when the flow shear scale length is less then the turbulent scale length of interest and the shearing rate is higher then the eddy turnover rate. In this case, the turbulent fluctuations are decorrelated by the shear more quickly then they would be by the turbulent interactions; consequently, the turbulent amplitude and scale lengths are reduced. This mechanism is very general and, assuming the sheared flow is stable and therefore not generating more turbulent fluctuations, should be a valid effect with all fluctuation models. The next and sometimes more important impact of the shear flow on the fluctuation amplitude is at the linear stabilization level. This effect is mode dependent and is therefore not as general as the nonlinear shear suppression, but for the modes on which it is effective there can be a significant impact. ${ }^{13}$ The mechanism is often straightforward, since the growth rate for many modes is $\omega$ dependent, the addition of shear can change $\omega$ and therefore change the growth rate or even completely stabilize the mode by raising the effective stability boundary. A third mechanism closely related to the first one is a direct effect on the transport. The previous two mechanisms reduced the turbulent transport by reducing the turbulence; this method can reduce the turbulent transport by changing the phase relationship between the advecting and advected fields. Because the transport comes from 
the cross correlation of two fields (i.e., $n$ and $\phi$ ), if the phase relationship between these fields is changed, the transport can be also. Sheared flow can have an effect on the average phase between the fields, thereby changing the transport. ${ }^{14,15}$

A final mechanism, which may be in some cases essentially the same as the first mechanism, by which sheared flow can have an impact on turbulent transport is the one that will be explored in the rest of this paper. If the transport takes place as correlated transport events, similar to avalanches in snow or sand, rather than as the sum of individual local transport, it is plausible to imagine that the sheared flow could decorrelate the long transport events. These correlated "avalanches" could be due to modulational interactions of the small-scale fluctuations ${ }^{16,17}$ or a simple sequential transport of some evolving field (temperature, density, etc.). The latter is very much like the traditional picture of a snow or sand avalanche, propagating and spreading after being initiated at one point. This is the model we will investigate.

\section{THE RUNNING SANDPILE MODEL}

Because of the expense and difficulty of accurately modeling large regions of a magnetic confinement device and because of the monumental task of dealing with and interpreting the data that one does get, it is often useful to construct the simplest model that captures the dynamics of interest. Starting from the assumptions of the importance of marginality to turbulent transport and the importance of turbulent transport to relaxation of gradients, a very simple natural model presents itself. In this model, local turbulent fluctuations are excited by the local gradient exceeding marginality, and the local fluctuations in turn relax the local gradient, transporting the excess gradient down the profile. This sandpile SOC model has the gradient modeled by the slope of the sandpile, while the turbulent transport is modeled by the local amount that falls (overturns) when the sandpile becomes locally unstable. The model system is driven by noise from the heating sources or background fluctuations, which in the sandpile model are represented by a 
random "rain" of sand grains on the pile. This model allows us to study the dynamics of the transport independent of the local instability mechanism and independent of the local transport mechanism. Because of the relative simplicity of the model, we are also able to do very long time calculations and collect reasonably large statistical samples.

A standard cellular automata algorithm ${ }^{18}$ is used to study the dynamics of the driven sandpile. The domain is divided into cells, which are evolved in steps. First, "sand grains" are added to the cells with a probability $P_{0}$. Next, all the cells are checked for stability against a simple stability rule and either flagged as stable or not, and finally, the cells are time advanced, with the unstable cells overturning and moving their excess "grains" to another cell with the size, distance, and direction of the fall being determined by the overturning rules. The most simple set of rules used is:

if

$$
Z_{n} \geq Z_{\text {crit }}
$$

than

$$
h_{n}=h_{n}-N_{f}
$$

and

$$
h_{n+1}=h_{n+1}+N_{f}
$$

With $h_{n}$ defined as the height of cell $n, Z_{n}$ being the difference between $h_{n}$ and $h_{n+1}, Z_{c r i t}$ is the critical gradient and $N_{f}$ is the amount of "sand" that falls in an overturning event (Fig. 1). In terms of the normal physical quantities we associate with turbulent systems, each cell can be thougint of as the location of a local turbulent fluctuation (eddy). $Z_{\text {crit }}$ is the critical gradient at which fluctuations are unstable and grow, and $N_{f}$ is the amount of "gradient" that is transported by a local fluctuation (local eddy-induced transport, for example). The average sandpile profile is equivalent to the mean temperature or density profile, while the total number of sand grains in the pile (the total mass) is the total energy/particle content of the device. The "sand grain" flux is clearly the particle/heat flux in the turbulent system, and for later reference, the sheared flow (wind) is the sheared electric field often coincident with transport barriers. 
The simulations are done in a two-dimensional system (slab geometry) where $x$ is equivalent to the radial coordinate $(r)$ and $y$ to the poloidal angle $(\theta)$. We have used a variety of domain sizes varying from $50 \times 1$ ( $x$ and $y$ directions) to $800 \times 100$ with most of the 2-D calculations being performed at $200 \times 50$. The boundary conditions for the computation domain are periodic in the $y$ direction, open at $x=L$ (particles that reach the edge are lost), and closed at $x=0$. Computations are typically started from a marginal state (i.e., $Z_{n}=Z_{\text {crit }}-1$ ) and allowed to relax to the steady state. The relaxation time is a function of $L, P_{0}$, and $N_{f}$, and an $L=200$ for typical values of $P_{0}$ and $N_{f}$ is around 40,000 time steps. To accumulate sufficient statistics, the system is iterated for $10^{5}$ to $10^{7}$ time steps after saturation is reached. The main diagnostic for the sandpile model avalanche dynamics is the time history of the number of flips (overturning events), with both the total number in the system and the number of flips for individual $y$ values tracked. Additionally, local and poloidally averaged particle fluxes are tracked at a few radial positions. Finally, the evolutions of both the total mass (the sum of all the grains in the system) and the average profiles are followed.

To investigate the normal SOC transport dynamics and provide control results with which to compare computations with sheared flow, we have reproduced the previously published sandpile computations ${ }^{2,3}$ with shear free running sandpiles. Since the model only has four parameters, $L, P_{0} L, N_{f}$, and $Z_{c r i t}$, we have performed scans of each of these parameters, keeping the others fixed. The results of these scans are summarized elsewhere. ${ }^{19}$ Below we review the results from a "typical" case and compare its dynamics to that of a marginal system. In our typical run, $L=200, P_{0}=0.0025, N_{f}=3$ and $Z_{\text {crit }}=$ 8. This run was started marginally stable $(Z=7)$ and then run $\sim 10^{5}$ steps into the SOC regime. The relaxation from the marginal profile to the $S O C$ profile can be seen in the time evolution of the number of flips (Fig. 2). The SOC state is not reached until the average level of the number of flips saturates at approximately 30,000 time steps. 
Figure 3 shows the marginal profile (from a system with the same parameters as the typical case but with $N_{f}=1$ ) and the average SOC profile, both averaged over the last 20,000 steps. It can be seen readily from the slope of the SOC profile and the number of flips occurring after relaxation into the SOC state that even with a significantly submarginal profile the system is able to robustly transport the inputted flux. This is an important characteristic of SOC systems and should lead to a reexamination of the relevance of some of the modes whose importance was discounted because the profiles were submarginal (i.e., ballooning modes). The one condition needed for the maintenance of a SOC profile rather than a marginal profile is that $N_{f}$ be greater than 1 . As discussed earlier, this is equivalent to saying that a turbulent eddy will attempt to transport enough to level the local gradient in one eddy turnover. If $N_{f}=1$, then whenever a sand grain is dropped onto the pile it will fall all the way down to the bottom of the pile and exit at the base. This fall is not an organized avalanche in the sense that it will not grow as it cascades down the pile because only the local cell with the extra grain is unstable (supermarginal). Comparing a time history of overturning sites in a marginal system $\left(N_{f}=1\right)$ with a time history of overturning site in our prototypical SOC case $\left(N_{f}=3\right)$ clearly shows the difference. In the marginal case [Fig. 4(a)], all of the falls are individual isolated events (except for the places where two sand grains were dropped by chance in neighboring cells), while in the SOC case [Fig. 4(b)] there clearly exist coherent avalanches of all different lengths. These figures are time histories of a given poloidal location with all the poloidal positions giving statistically the same result. The dark cells are cells that are overturning at that time step while the light cells are stable. It can be seen from the diagonal lines of overturning sites that the transport in the marginal case is continuous from the point of input (the location of the random grain-drop) to the bottom edge where the grain exits the system. In the SOC case, coherent avalanches can be seen to grow and shrink. Some are seen to propagate up the slope, while others propagate down or in both directions. It should be kept in mind that the flux always moves down, for these automata rules. The upward propagation is really a 
void moving up, which is by symmetry the same as a bump moving down. This dual propagation is a signature of the SOC system. Even though both cases are in steady state, meaning that the flux in via the random rain of grains is the same as the flux out at the bottom, there is clearly a more bursty character to the SOC case with the flux often exiting in coherent avalanches rather than the continuous single transport events that make up the marginal system. To quantify the distribution of these avalanche events that typify the transport dynamics of the flowing sandpile, we analyze the avalanches in a number of different ways.

First and most simply, one can construct the probability distribution function (PDF) for the total number of instantaneous flips (overturning event). This is explored in more detail in Ref. 19. The PDF for the SOC case has a mean given by the flux into the system, $P_{0} L$, times the average time for the grains to leave the system, $L / 2$, weighted with the amount transported in one flip, $N_{f}$, giving a mean of $P_{0} L^{2} / 2 N_{f}$. The variance for the SOC cases seems to scale with the mean as one would expect from a Poisson distribution. Because of the need for very large samples to quantify the higher order moments of the PDF in which differences often appear, a better method for differentiating between the dynamics is the correlation function. The auto-correlation function can give information about the average length of the avalanches (transport events), while cross-correlation functions between radially separated points quantifies the radial correlation length of these events. The average length of the avalanche (the width of the auto-correlation) is seen to be $\sim 1$ in the marginal case, but in the SOC case it is $\sim 8$ (Fig. 5). The radial correlation length is found to be $\sim 14$ in our typical SOC case [Fig. 6(a)]. Recalling that the individual cell represents the local fluctuation, the relatively long correlation length signifies much longer transport correlation lengths than fluctuation correlation lengths. This feature of SOC dynamics is borne out in a model realization using a 3-D resistive pressure-gradient-driven turbulence model. The results of this realization will be presented elsewhere. The marginal case has even longer radial correlation lengths because of the nature of a transport event. In 
the marginal system, once a transport is started it continues all the way to the bottom. Therefore, we need another method to separate the marginal system from the SOC system. This can be done easily using the structure of the cross-correlation function. Remembering that the SOC system avalanches propagated both up and down while the marginal system only propagated down, the cross-correlation function between radially separated points can be expected to have a peak at the lag given by the separation of the radial points (assuming one radial step in one time step). The marginal case will have this peak only on the lag side of the correlation function, while the SOC case will have a peak on both the lag side, signifying downward propagating avalanches, and the lead side, signifying upward propagation [Fig. 6(b)]. The relative heights of these two peaks give the local ratio of upward to downward propagation and may be used as a local measure of the profile's deviation from marginal. These quantities depend weakly on the flux through the given radial location, which is in this case simply $P_{0} x$; this leads to a radial dependence of the auto and cross-correlation functions in the SOC system.

The third method for quantifying the avalanche dynamics is with frequency diagnostics applied to time history of instantaneous flips (only using the saturated region). A typical spectrum can be divided into three regions following Hwa et al. ${ }^{3}$ (Fig. 7). The first region is the high frequency end of the spectrum, which follows approximately an $\omega^{-4}$ power law. This region is identified as the noninteracting (or overlapping) avalanche region. If $P_{0}$ is made small enough, this region completely envelops Region 2 (middle to low frequencies), which is identified as the overlapping avalanche region. The spectral falloff in the overlapping region is approximately $\omega^{-1}$, and this is the region of primary interest to us. Finally, there is Region 3, the lowest frequencies. In this region the spectral power is relatively flat and finally rolls over at the lowest frequencies. This region is identified with global discharge events that have extremely long correlation times. It is easiest to see these discharge events by looking at the time history of the total mass (the integrated heights). Figure 8 shows the total mass in a case that was run for $10^{7}$ time steps 
and encompasses perhaps two of the largest "global discharge events." Note that the frequency of these largest events is proportional to the rain rate (the input flux) as one would expect for relaxation-type oscillations because the rate of refilling of the pile after a massive discharge is proportional to the input flux. Region 3 is a very interesting regime dynamically and is the region of primary interest to those using SOC models to study earthquakes. However, because this region involves time scales probably much longer than a confinement time (which is on the order of $L^{2}$, assuming transport goes one fluctuation size in one time step), we will only explore the high-frequency end of this region.

To compare these cellular automata model results with analytic results, ${ }^{1}$ we construct a diffusion coefficient $D_{0} . D_{0}$ is built in the typical fashion from the average local flux and average local gradient, giving $D_{0}=\langle B /<d h / d x\rangle$. However, since the system is in steady state, the average local flux through $x_{0}$ is simply the average number of grains falling into the region above $x_{0}$, which is given by $P_{0} x_{0}$. This then allows us to write $D_{0}$ as $D_{0}=P_{0} x_{0} /<d h / d x>$. It is found that $\left\langle d h / d x>\right.$ scales with $P_{0} x$, allowing a natural way to look at the diffusion by plotting $D_{0}$ against $P_{0} x$, which is shown in Fig. 9. It is found that $D_{0}$ has a functional dependence of $\left(P_{0} x\right)^{\beta}$ with $\beta \approx 0.95$ in the region where $P_{0} x$ is less than $N_{f} / 2$. This "fast" transport which scales with the system size is in agreement with the analytic work by Diamond and Hahm on the Burgers equation. Very fast diffusion or ballistic propagation is also observed in the 3-D resistive pressure-gradient driven turbulence model realization of a SOC system. These results will be published elsewhere.

\section{SHEARED SOC MODEL}

Into the basic model described above we now add a region of poloidal sheared flow. This is implemented by adding a constant flow in one direction to the top of the sandpile and a constant flow in the other direction to the bottom. The two constant flow regions are then connected by a shear flow region (Fig. 10) with a variety of possible 
shear profiles. The shear is defined as the velocity increment, $\Delta V$, between two adjacent cells in the $x$ direction. The poloidal flow is added to the dynamics in the time advance step after moving any falling grains to their new positions. The impact of the shear flow is quantified by changing a shear parameter, $S$, equal to $\Delta V$ times the size of the shear region, $L_{S}\left(S=L_{S} \Delta V\right)$.

The effect of the sheared flow on the transport dynamics can be first and most easily observed in a time history of the overturning sites [Fig. 11(a)]. The sheared flow region in the middle is easily differentiated from the unsheared ends by the absence of correlated transport events (avalanches) in the shear zone. This can be contrasted to the unsheared SOC case shown in Fig. 11(b). While the difference in avalanche dynamics is visually striking and shows clearly the decorrelation of the long transport events by the sheared flow, to quantify the changes we must use the other diagnostics. The PDF of the flips displays a marked decrease in the variance for a running sandpile with flow shear when compared to one without shear. This trend continues when the shearing rate and/or the size of the shear zone increases. This suggests that the larger scale transport events are being suppressed by the sheared flow and, since the total flux must remain the same, the medium- and small-scale events must increase to make up the difference. The frequency spectra make an even more compelling case for the impact of sheared flow on the transport dynamics of the running sandpile. Simply comparing the spectra for an unsheared case with a sheared case (Fig. 12), one can see a suppression of the low frequency end of the spectrum and an increase in the high frequency end. This can be quantified through the mean frequency $\varpi$, defined as $\varpi=\int \omega S(E) d \omega$. Figure 12(b) shows the variation in $\omega$ as the shear parameter is increased. This shows the decorrelation time of the transport decreasing as the shear parameter increases. Once again it is important to note that this effect is completely different from the shear suppression of turbulence. In this model the turbulent amplitude and stability boundry are not being affected at all; it is only the correlated transport events that are being modified. Therefore, this decorrelation time is not 
the standard turbulent decorrelation time but rather a new quantity, a transport decorrelation time. In the shear free case, the transport decorrelation time is longer than $L^{2}$, while in the sheared flow case, the decorrelation time becomes shorter than $L^{2}$.

The next logical questions to ask are, (1) if the transport events are being decorrelated, what is the impact on the diffusion coefficient; and (2) does this build up a transport barrier? The answers to both of these questions are somewhat subtle. In the sheared flow region, there is a substantial change in the functional form of the steady state sand pile slope. Therefore, the diffusion coefficient $D_{\text {eff }}$ changes functional form, leading to an increase in the diffusion coefficient for small $P_{0} x$ and a decrease for large $P_{0 x}$. Again, we find a universal curve for $\Gamma /\langle d h / d x\rangle$ when plotted versus $P_{0} x$ (Fig. 9). In this case, the slope of $h$ cannot be described by a power function, but it is just a linear function of $P_{0} x$. As a consequence, the effective diffusion coefficient is

$$
D_{e f f}=\frac{P_{0} x}{a+b P_{0} x}
$$

Asymptotically, for $x \rightarrow \infty, D_{\text {eff }} \rightarrow b^{-1}$, which becomes independent of $x$. For $N_{f}=3$ and $\Delta V=1$, a fit to all the data gives $a=4.85$ and $b=1.59$. This change in functional form is consistent with the change in dynamics predicted by analytic work on the Burgers equation model by Diamond and Hahm. 1 The analytic form of the diffusion coefficient goes from infrared divergent $\left(D \propto k_{r}^{-1}\right)$ in the shear free case to independent of $k_{r}\left(D \propto k_{r}{ }^{0}\right)$ in the sheared flow case. The asymptotic limit $(x \rightarrow \infty)$ of the diffusion coefficients in the sand pile model shows the same dependence going to a constant with shear and $k_{r}^{-0.98}$ without.

Due to the discrete nature of the system, the impact of increasing $\Delta V$ saturates when $\Delta \mathrm{V}$ is larger than one. This is because when $\Delta \mathrm{V}$ is larger than one all avalanches down to the cell size are decorrelated. Strong sheared flow with a given scale length will decorrelate all transport events with a larger scale length. Therefore, if the shear scale length is made 
smaller than the smallest transport event (which would be unphysical in a continuous turbulent system), all the avalanches would be decorrelated. Because of this effect, the method used to investigate the dependence of the asymptotic $D_{\text {eff }}$ on the shearing rate was to decrease $\Delta V$ below 1 . The effective asymptotic diffusivity is found to decrease (Fig. 13) with increasing shear as $D_{\text {eff }} \propto(\Delta V)^{-0.51}$. This is in comparison to the analytic form from the Burgers equation model by Diamond and Hahm, which gives a $D_{\text {eff }}$ dependence on $\Delta V$ with $\gamma=4 / 5$. While the coefficients are not the same, given the differences in the models (one being continuous the other being discrete), the similarity in scalings is remarkable.

In the model as presented up to this point, the inclusion of sheared flow either can cause a transport barrier, a steepening of the gradient with the coincident decrease in the diffusion coefficient, or an anti-transport barrier in which the gradient is further reduced and the diffusion coefficient (as defined) is therefore increased. Which occurs depends on the ratio of $N_{f}$ to $P_{0} x$; when $N_{f} P_{0} x$ approaches 3 (for typical parameters), the diffusion coefficient crosses the shear-free SOC coefficient (Fig. 9) and a transport barrier is formed. It should be kept in mind that the two other effects of shear on turbulent transport, the shear suppression of turbulence and the increased stability of the mode, are not included in this model. These will be dealt with briefly later.

As mentioned before, most of these results are for cases in which $P_{0 x}$ does not exceed $N_{f} / 2$ anywhere in the computational domain. The reason for this is to enable the unambiguous study of the SOC dynamics. When the average local flux exceeds $N_{f} / 2$, a distinct change in the dynamics occurs. The average local gradient jumps from the "submarginal" SOC gradient to a super-marginal gradient. This jump is coincident with a region in which the avalanches are occurring almost constantly as would be implied by the super-marginal gradient. This region allows for a natural definition of an edge zone and has interesting implications for the initiation of the transport barrier at the edge in an $\mathrm{L}-\mathrm{H}$ transition. Further investigation of this area will be published later, but it should be noted 
that in this edge region, shear increases the gradient further, creating a transport barrier that forces a large pedestal to develop inside.

As discussed before, sheared flow can have multiple effects on turbulent transport. By using the simple automata model, one is able to isolate the effect of the shear decorrelation on the transport events from any other stability effects. Those other effects can be added to the simple model in an ad hoc manner. In the sand pile model, the overturning events are analogous to the turbulent fluctuations driving the transport; therefore, since sheared flow can reduce the turbulence amplitude, in the sandpile paradigm $N_{f}$, the amount moved in an overturning should be affected by the shear. Similarly, because sheared flow often has an effect on the linear stability of a mode, shear should have an impact on $Z_{c r i t}$, the marginal stability level. If we include in the sandpile model a reduction in $N_{f}$ proportional to the shearing rate and an increase in $Z_{c r i t}$ also proportional to the shearing rate, we obtain a confluence of effects on the gradient, and therefore on the diffusion also, due to the sheared flow that add up to more than the sum of the individual changes (Fig. 14). This is mainly due to the increased avalanche rate needed to maintain the flux when $N_{f}$ is decreased. This then forces the sheared region to be more "edge" like, causing the development of a large transport barrier. The inclusion of these two additional effects is not meant to be self-consistent but rather is a demonstration that the real impact of shear on transport is likely to be enhanced by the combination of effects.

\section{CONCLUSIONS}

Within the constraints of a simple cellular automata model of critical gradient dynamics (the running sandpile model), it is found that:

1) Robust transport can occur in a noise driven system with profiles that are on average submarginal. This may be relevant to the experimental observation that over much of the radius the profile appears to be marginal or submarginal to most of the instabilities suspected of dominating transport. 
2) Transport events, avalanches, are found on all size and time scales in the running system. The coherence of large transport events can make the transport scale with the system size (Bohm-like scaling), even though the local transport mechanism is the much smaller scale local fluctuations. Additionally, the dominance of the large avalanches in the transport dynamics make the flux very temporally bursty.

3) The addition of sheared flow to the running sandpile has a fundamental impact on the transport dynamics. The dominant transport scales move from system size to smaller scales with the diffusion no longer being infra-red divergent $\left(k^{-1}\right)$. The mechanism for this change is the simple decorrelation of the large scale transport events by the sheared flow.

4) With moderately strong driving (or in the limit of large $P_{0 x}$ ), the inclusion of shear can cause the formation of a "transport barrier" (a region with decreased diffusivity) consistent with theoretical predictions. However, in this model, which does not include the standard sheared flow effects such as linear stabilization, very weak driving can lead to an increased diffusivity in the sheared flow region. When the other impacts of shear are included in an ad hoc manner, the shear region always exhibits a decreased diffusivity with the coincident transport barrier.

The possibility of transport that is largely independent of the nature of the local instability and furthermore can occur even with average gradients that are submarginal should lead to the reevaluation of some modes that may have been discounted due to the stability of the profiles. Because the dynamics of the transport are not closely tied to the local dynamics (the instability driving the fluctuations), this type of model would suggest a universality in transport even when the instabilities are different (i.e., across machines). The discrepancy between the apparent observed Bohm scaling in experiments and the 
Gyro-Bohm scaling predicted by most turbulence theories may be addressed by this universality in the transport dynamics independent of the instability.

Finally, the change in dominant scales in the sandpile model with the addition of shear is not inconsistent with the experimental observation that transport scalings seem to go from Bohm to Gyro-Bohm when the system goes into the enhanced confinement mode, which has flow shear. While it is unlikely that this decorrelation of transport events is the entire answer, it does provide interesting avenues to explore both experimentally and theoretically.

\section{ACKNOWLEDGMENTS}

Valuable discussions with J.-N. Leboeuf are gratefully acknowledged. D. E. Newman would like to thank Oak Ridge National Laboratory for its support through the Wigner Fellowship program. This work is supported by the U. S. Department of Energy under contract DE-AC05-84OR21400 with Lockheed Martin Energy Systems, Inc., and Grant No. DE-FG03-88ER-53275 with University of California, San Diego. 


\begin{tabular}{|c|c|}
\hline $\begin{array}{ll}1 . & \begin{array}{l}\text { Turbulent Transport in } \\
\text { Toroidal Plasmas }\end{array} \\
\end{array}$ & Sandpile Model \\
\hline Localized Fluctuation (eddy) & Grid Site (cell) \\
\hline $\begin{array}{l}\text { 5. Local turbulence mechanism: } \\
\text { 6. Critical gradient for local instability } \\
\text { 7. Local eddy-induced transport }\end{array}$ & $\begin{array}{l}\text { 8. } \quad \text { Automata Rules: } \\
\text { 9. } \quad \text { Critical sandpile slope }\left(Z_{c r i t}\right) \\
\text { 10. } \\
\text { Number of grains moved if unstable } \\
\left(N_{f}\right)\end{array}$ \\
\hline 11. Total energy/particle content & Total number of grains (total mass) \\
\hline $\begin{array}{l}\text { 13. Heating noise/background } \\
\text { fluctuations }\end{array}$ & Random rain of grains \\
\hline 15. Energy/particle flux & Sand flux \\
\hline Mean temperature/density profiles & Average slope of sandpile \\
\hline 19. Transport event & Avalanche \\
\hline 21. sheared electric field & 22. sheared flow(sheared wind) \\
\hline
\end{tabular}

Table 1 


\section{FIGURE CAPTIONS}

Fig. 1 A cartoon representation of the simple cellular automata rules used to model the sandpile.

Fig. 2 Time history of the total number of overturning sites at each instant. The figure shows the relaxation from the marginal profile in the first 30,000 time steps followed by the evolution within the SOC state.

Fig. 3 The average sandpile profiles for a marginal case and a SOC case. For both cases $Z_{\text {crit }}=8$ and both cases are transporting the same number of grains.

Fig. 4 A visualization of the overturning sites at all radiuses at one $\theta$ location as a function of time. The bottom of the figure is the bottom edge of the sandpile. Light colored cells are stable while dark cells are the unstable (overturning) sites. a) Shows the avalanches for a marginal case. All the grains that fall onto the sandpile move down the pile singly exiting at the bottom. This can be seen by the diagonal lines angling down as time increases. b) Shows the avalanches in a marginal case. The avalanches can be seen to grow to radial sizes and last various lengths of time. The propagation of the disturbance can be seen to move both up and down the slope.

Fig. 5 The auto-correlation functions for a marginal system having a width of about 1 and a SOC system having a width of about 10 . These widths show the difference in avalanche lengths between the marginal and SOC systems.

Fig. 6 a) The radial correlation length of the flux is given by the half height of the falloff of the cross-correlation functions with radial separation. 
b) A typical cross-correlation function for $\Delta r=40$ cells. Note the double peaks at both a lag and lead of 40 (the separation distance). These are due to the dual propagation nature (both up and down) of the SOC avalanches.

Fig. 7 An autopower frequency spectrum of the flips in the saturated SOC state. This typical sandpile frequency spectrum shows the 3 dynamical regimes.

Fig. 8 The time evolution of the total mass (the space integrated number of grains) displays less of the high frequency oscillations then the "flips" but more low frequency oscillations. Note that in $2 \times 10^{7}$ time steps there are only one or 2 of the largest relaxation type oscillations.

Fig. 9 The diffusion coefficent as a function of flux for a case with sheared flow and one without sheared flow. Note the different functional forms.

Fig. 10 A cartoon of the sandpile with a shear flow zone. The whole pile is flowing to the right at the top and to the left at the bottom connected by a variable sized region of sheared flow.

Fig. 11 Time evolution of the overturning sites (like figure 4). The avalanches do not appear continous in time because only every 50th time step is shown. a) The shear free case shows avalanches of all lengths over the entire radius. b) The case with sheared flow shows the coherent avalanches being decorrelated in the shear zone in the middle of the pile.

Fig. 12 a) Frequency spectra with and without a shear flow region. This shows a marked decrease in the low frequency power (with shear) and a commencerate increase in high frequency power. 
b) The insert shows the decorrelation time $\left(\tau_{d}=1 / \varpi\right)$ as a function of the shear parameter (the product of the shearing rate and the size of the shear zone).

Fig. 13 The diffusion coefficent as a function of the shearing rate.

Fig. 14 The slopes of a sandpile with a shear region in the middle including all the shear effects (diamonds) and just the transport decorrelation and the linear effect (circles). 


\section{REFERENCES}

1 P. H. Diamond and T. S. Hahm, On the dynamics of turbulent transport near marginal stability, Physics of Plasmas, in press.

2P. Bak, C. Tang, and K. Wiesenfeld, Phys. Rev Lett. 59, 381 (1987).

${ }^{3}$ T Hwa and M. Kardar, Phys. Rev A 45, 7002 (1992).

4J. M. Carison and J. S. Langer, Phys. Rev. Lett. 62, 2632 (1989).

5P. C. Liewer, Nucl. Fusion 25, 543 (1985).

6.J. W. Connor, J. B. Taylor, and M. Turner, Nucl. Fusion 22, 256 (1984).

7T. S. Hahm and W. M. Tang, Phys. Fluids B 1, 1185 (1989).

${ }^{8}$ H. Biglari, P. H. Diamond, and M. N. Rosenbluth, Phys. Fluids B 1, 109 (1989).

9G. W. Rewoldt, L. Lao, and W. M. Tang, paper 3C48 Sherwood, 1995.

10F. W. Perkins et al, Phys. Fluids B 5, 477 (1993).

${ }^{11}$ T. C. Luce et al, in Proceedings of Fifteenth International Conference on Plasma Phys. and Contr. Fus. Research, Seville, Spain, Paper A-2-111-2 (1994).

12H. Biglari, P. H. Diamond, and P. W. Terry, Phys. Fluids B 2, 1 (1990).

13B. A. Carreras, K. Sidikman, P. H. Diamond, P. W. Terry, and L. Garcia, Phys. Fluids B 4, 10 (1992).

14B. A. Carreras, V. E. Lynch, L. Garcia, and P. H. Diamond, Dynamics of Second Order Phase Transitions in Resistive Pressure-Gradient-Driven Turbulence, Phys.

Plasmas, in press.

15R. A Moyer et al; Phys. Plasmas 2, 2397 (1995).

16V.D. Shapiro et al; Comments on Plasma Physics and Nuclear Fusion, 16, 155 (1995).

${ }^{17}$ V. B. Lebedev, P. H. Diamond, V. E. Shapiro and G. I. Soloviev; Physics of Plasmas, in press (1995).

18L. P. Kadanoff, S. R. Nagel, L. Wu, and S-M. Zhou, Phys. Rev. A 39, 6524 (1989). 
${ }^{19}$ D. E. Newman, B. A. Carreras and P. H. Diamond; Proceedings of the 2 nd Conferance

on Transport, Chaos and Plasma Physics, to be published by Word Scientific

\section{DISCLAIMER}

This report was prepared as an account of work sponsored by an agency of the United States Government. Neither the United States Government nor any agency thereof, nor any of their employees, makes any warranty, express or implied, or assumes any legal liability or responsibility for the accuracy, completeness, or usefulness of any information, apparatus, product, or process disclosed, or represents that its use would not infringe privately owned rights. Reference herein to any specific commercial product, process, or service by trade name, trademark, manufacturer, or otherwise does not necessarily constitute or imply its endorsement, recom-

/ mendation, or favoring by the United States Government or any agency thereof. The views and opinions of authors expressed herein do not necessarily state or reflect those of the United States Government or any agency thereof. 